\title{
Kaisipang Sosyalismo sa mga Akda ni Amado V. Hernandez
}

Ferdinand Tablan

\begin{abstract}
The aim of this paper is to trace the root of socialistic thought in the Philippines after the Second World War. As such, the novels, poems, essays, and short stories of Amado V. Hernandez are examined. Hernandez is considered as one of the foremost socialist writers during the 1950's to 1970's - a poet, activist, leader of the labor movement, and a National Artist. In order to understand the emergence of socialistic thinking in the Philippines, the situation of the country from 1946-1970 is examined; this will determine whether socialism in the Philippines is, indeed, influenced by Mao and Lenin. Despite the poetic or literary style of Hernandez's writings, it is still possible to reconstruct the socialist theme in his writings through a historico-philosophical approach.
\end{abstract}

Key words: Amado V. Hernandez, socialism, Marxism, Filipino philosophy

$\mathrm{K}$

aramihan sa mga importanteng naisulat ni Amado V. Hernandez na mababasa pa sa kasalukuyan ay nasa anyong tula o kwento kung saan pananaw at ideya. Sa pamamagitan ng pamamaraang historikal at pilosopikal, nais ng pag-aaral na ito na maipaliwanag at mailahad ng tuwiran at sistematikong paraan ang kaisipang sosyalismo sa mga akda ng nasabing autor.

Ayon kay Karl Marx,

The totality of these relations of production constitutes the economic structure of society, the real foundation on which arises a legal, political, superstructure and to which correspond definite forms of social consciousness. The mode of production of material life conditions the general process of social, political, and intellectual life.

Tunay ngang ang literatura ay kathang-isip lamang at kahit pa ito ay naglalaman ng mga bagay na tumutukoy sa buhay at panahon ng may-akda, ang mga ito'y bahagi pa rin ng obra at dapat tratuhin na gayon. Ngunit ito'y hindi nangangahulugang ang historikal o pilosopikal na pag-aaral ng panitikan ay

${ }^{1}$ Karl Marx, Early Writings, trans. by Rodney Livingstone and Gregor Benton (New York: Vintage Books, 1975), 425.

(c) 2011 Ferdinand Tablan

http://www.kritike.org/journal/issue 9/tablan june2011.pdf ISSN 1908-7330 
walang saysay. Ang kamalayan ng manunulat o makata man ay sumasalamin sa kalagayan ng kanyang bansa at kapaligirang ginagalawan. Katulad ng economiya, politika, at iba pang uri ng sining, ang literatura ay nag-uugat sa lipinunan at hindi maaaring ihiwalay dito. Bagamat ang paglalahad ng ating kasaysayan ay gawain ng nga mananalaysay, ang mga nobelista at makata, tulad ni Hernandez ay gumagawa din nito sa paraang imaginative reconstruction. ${ }^{2}$ Ang panitikan ay maituturing din na kasaysayan, hindi lamang dahil ang akda ay naisulat sa lumipas na panahon, bagkus ay ipinapaloob nito ang kasaysayan sa paglalahad mismo. Ang wika ni Pantoja-Hidalgo hinggil sa mga historical novels:

History bere is not setting. It enters into the motivation of the characters; it propels the plot. The characters in these novels are political beings, their conflicts are engendered by political events. I would even claim that the real protagonist here is the nation itself; the real conflict, its desperate struggle for survival. ${ }^{3}$

Si Ka Amado mismo ang nagsabi na "Ang panitikan ay mga kathang makasining, taglay ang mataas an uri, na naglalarawan ng buhay, kaugalian, mga karanasan at kalagayan ng bayan o sosyedad sa isang kapanahunan, at lalong kanais-nais kung naranasan o nasaksihan ng awtor."4 Pinuna niya ang pagkahilig ng mga Pilipino sa mga romantisismo at eskapismo. Ang higit na mataas na uri ng katha ay "ang makinis na kombinasyon ng realismo at arte." 5

Naniniwala si Hernandez na ang manunulat ay dapat magkaroon ng kaugnayan sa kanyang kapanahunan, hindi tulad ng isang kaputol na kahoy na ibinalibag sa dagat at tinatangay ng alon. Sa kanyang aklat na Isang Dipang Langit, sinulat niya na siya ay sumasang-ayon sa paniniwala ni Salvador P. Lopez na, "The Filipino writer must grow up... He must write of virile people winning victories towards freedom, or of emaciated human beings enfeebled by anti-buman civilization. In short, he must write a red-blooded literature." 6 Dapat ang manunulat ay manaog sa kanyang toreng garing at yumapak sa lupa, pagkat ang arte ay kakambal ng tao at lupa ang bumubuhay dito. "Maaaring ang artista, manunulat at makata'y sumuko at manahimik upang mabuhay, ngunit ang ganya'y tiyak na kamatayan ng kanyang sining."” Sa kanyang palagay, tatlong bagay ang dapat taglayin ng sino mang mga mangangatha. Ito ay:

Una, kamulatang panlipunan o social consciousness na katambal ang diwa ng nasyonalismo.

\footnotetext{
${ }^{2}$ Christina Pantoja-Hidalgo, "History As Literature," in Coming Home (Pasig City: Anvil Press, 1997), 133.

${ }^{3}$ Ibid., 129 1997), 241

4 Amado V. Hernandez, Magkabilang Mukha ng Isang Bagol ( Quezon City: UP Press,

5 Ibid., 250.

3.

${ }^{6}$ Amado V. Hernandez, Isang Dipang Langit (Manila: Makabayan News Feature, 1961),

${ }^{7}$ Hernandez, Magkabilang Mukha ng Isang Bagol, 247.
} 
Ikalawa, diwa ang pakikibaka at kaunlaran upang maging mabisang kawal ng panitikan.

Ikatlo, dapat silang magbuklod sa isang malakas na katipunan upang makapanindigan, magkaroon ng boses sa pangangalaga sa kanilang kalayaan at karapatan at nang hindi sa habang panahong hamak silang upahan na ang trato ng mga magasing komersiyal at ng mga publisher ay mabuti pa ang trato sa isang labandera. ${ }^{8}$

Samakatuwid, ang pagsulat ay dapat may layuning panlipunan. Sa halip na panaginip o pantasya, ang sino mang mangangatha ay dapat sumulat base sa kanyang pag-iisip at kamalayan na naka-angkla sa kanyang kapaligirang ginagalawan. Si Hernandez ay sumulat, hindi lamang tungkol sa pag-ibig o mga pangarap kundi tungkol din sa mga manggagawa, hustisya sosyal, korapsyon sa gobyerno, pulitika, digmaan, kaapihan, kalayaan, bigas, welga, bartolina, gutom - walang bagay para sa kanya ang hindi maaaring paksain ng tula. Maging ang kanyang mga nobela at maikling kwento ay naglalarawan ng pang-araw-araw na buhay ng mga karaniwang tao tulad ng bombero "Sa Oras ng Panganib" Liwayway 1928), boksingero ("Ang Aguinaldo ng kanyang Anak" Liwayway 1929), piping katiwala na nagpakita ng kabayanihan ("Panata ni Pilar" 1936), peryodista at nurse ("Kulang sa Dilig" 1939), magsasaka ("Nagsilbi si Pastor" at "Supling ni San Husep" Liwayway 1959), simpleng buhay sa barrio ("Hindi Sumalubong is Major" 1959), unyonista ("Panata ng Isang Lider" Liwayway 1960), tindera ng sweepstakes ("Hubad" Liwayway 1958), at mag-asawang iskuwater na walang permanenteng trabaho ("Isang Ulo ng Litson" Liwayway 1959). Sa kwentong “Langaw sa Isang Basong Gatas" (Panata sa Kalayaan 1970), inilahad niya ang napakalayong agwat ng mga mahihirap at mayayaman. Isang pamayanan ng mga mahihirap ang pinilit na paalisin dahil sa nakasisira sila ng tanawin sa bagong katatayong exclusive subdivision. Problemang agraryo naman ang tema ng nobelang Luba ng Buwaya (unang nalimbag noong 1963). Sa tulangkasaysayang Bayang Malaya (1969), kanyang ipinakita ang kanyang malasakit sa karapatan ng mga maliliit. Nang mahalal si Lantay bilang pangulo ng unyon, ito ang laman ng kanyang talumpati:

Habang ang paggawa'y nagkakanya-kanya

Marami sa bilang, pakyaw ang halaga

Alam nating lahat

Sa anomang tibay ng iisang hibla

Mapapatid agad

Hindi kung yari nang lubid na abaka. (4416)

Walang komunismo sa lupaing tangi

Kung ukol sa lahat ang ani at yari,

Walang komunismo 
Sa bayang malaya at di nakatali

Ang diwa ng tao,

Komunismo'y apo ng masamang hari.(4446)

Kung ang kaisaha'y tunay na matatag

Ay bisig ng api't tinig ng mahirap,

Sila'y maririnig,

At magkakatimbang ang dalawang lakas,

Ang puhuna't bisig

Magkakausap na nang pantay at patas.(4458)

Si Hernandez ay isinilang sa Tondo noong Setyembre 13, 1903. Dito siya nagkaisip at lumaki. Isang tula ang kanyang isinulat tungkol sa Tondo na kanyang inilarawan bilang isang lugar na mahirap at matao.Tondo ay lungsod ng mga makata at bayani kung saan nagmula sina Raha Soliman, Martin de Goiti, Balagtas, at Huseng Sisiw. Ang panahon ni Hernandez (1903-1970) ay sumasakop sa panahon ng pananakop sa bansa ng mga Amerikano, panahon ng Commonwealth, ikalawang digmaang pandaigdig, at ng unang tatlong dekada ng panahon ng kalayaan. Ang pananankop ng Hapon ay lalong nagpaigting sa relasyon ng mga Pilipino at mga Amerikano habang sila'y nagtulong sa digmaan. Dahil din sa digmaan, maraming mga maylupa ang napilitang iwanan ang kanilang hacienda at ito ay napunta sa kamay ng mga magsasaka na nakaranas ng kalayaan at pagsasarili. Nagtatag sila ng Hukbalahap. Ngunit matapos ay digmaan ay pinaalis sila sa lupang kanilang sinasaka at itinuring ni Pangulong Roxas na tulisan dahil sa pagsuporta nila sa kalaban sa pagkapangulo na si Osmena. Malungkot na winika ni Hernandez na ang nangyari sa Pilipinas ay kabaliktaran sa naganap sa mga bansa sa Europa kung saan ang mga bayaning nakipaglaban sa mga mananakop ang siyang pinaranglan at humawak ng liderato matapos ang digmaan. Sa halip, ang mga bayani ay inusig at tinugis. Maraming suliranin ang kinaharap ng bayan matapos ang digmaan tulad halimbawa ng mga isyung kolaborasyon at back-pay, rehabilitasyon, at kahirapan.

Sa lahat halos ng bansa sa Europa na sumailalim sa pagsakop ng mga Nazi ay nagpairal ng malalaking pagbabagong panlipunan pagkaraang makamit ang tagumpay, lalo na sa pakikitungo sa mga magbubukid, at mabilis na nagpasok ng mga makabagong paraan at palakad sa pagsasaka. Ngunit sa Pilipinas, ang mga bagaybagay ay ibinalik kapagkaraka sa dating kalagayan noong hindi pa nagkakadigmaan. ${ }^{9}$

Bagamat kinilala ng mga Amerikano ang kalayaan ng Pilipinas, ang konstitusyon naman ay inamendahan upang mabigyang daan ang Parity Clause ng Bell Trade Relations Act na nagbibigay ng karapatan sa mga Amerikano na

${ }_{9}^{9}$ Amado V. Hernandez, Progressive Philippines (Manila: Pilipino Press, 1949), 22. 
gamitin ang likas na yaman ng ating bansa. Nagkaroon din ng Military Bases Agreement sa pagitan ng Pililpinas at Amerika. Tinutulan ni Amado Hernandez ang mga patakarang nabanggit. Ngunit iilan lamang ang nakauunawa sa masamang epekto ng mga ito. Para sa mga nakararami, kaibigan natin ang mga Amerikano at nasa kamay nila ang ating proteksyon at pag-unlad. Hindi nagtagal ay unti-unting lumitaw ang mga suliranin sa politika at ekonomiya. Ang sistemang piyudalismo ay nagpatuloy, kontrolado ng mga Intsik ang pagtitingi ng mga bilihin, mga Amerikano naman ang sa malalaking korporasyon.Nagkaroon ng mga smuggling, kawalan ng trabaho, kahirapan at inflation. Ang kongreso na kontrolado ng mga maylupa at mayayaman ay pilit hinahadlangan ang anumang reporma sa lupa. Hindi naisagawa ang industrialisasyon. Ang alitan o cold-war sa pagitan ng Estados Unidos at Unyong Subyet matapos ang ikalawang digmaang pandaigdig at paglaganap ng komunismo sa Asya ay nagdulot ng pangamba sa maraming tao upang maging mapaghinala sa ano mang progresibong kilusan na maaaring ginagatungan ng mga komunista. Isang halimbawa ay ang pag-outlaw ni Presidente Roxas sa Pambansang Kaisahan ng mga Magsasaka.

Nagsimula si Ka Amado bilang isang kolumnista sa pahayagan, taong 1926. Sa gulang na 28 ay naging pinakabatang patnugot ng pahayagang Mabubay. Naging patnugot din siya ng Sampaguita, Filipino, Makabayan, at Ang Masa kung saan nakilala siya sa kanyang pagsuporta sa independence movement at mga militanteng pananaw. Sa kabila ang kanyang mga sulatin, nakilahok din siya sa iba't-ibang kalupunan ng mga manunulat, manggagawa, mahihirap at mga cause-oriented groups tulad ng Writers and Artists Sector: Movement for the Advancement of Nationalism, Pambansang Kombensyon ng Partido Nasyonalista, Democratic Labor Party, Civil Liberties Union, Congress of Labor Organization, at iba pa. Nanungkulan siya bilang konsehal ng Maynila sa loob ng dalawang termino (1945-46; 1948-51) hanggang siya ay ipakulong ng pamahalaan. Enero 26, 1951, matapos ma-raid ng militar ang punong tanggapan ng Civil Liberties Union ay inaresto si Hernandez sa salang rebellion complex with arson, murder, and robbery. Hinatulan siyang mabilanggo ng habang buhay noong Marso 1956. Ayon kay San Juan "In the atmosphere of McCarthyism, bigotry and official corruption then prevailing, he was arrested and accused of rebellion against the established government... he was convicted in spite of the testimony of respected civic leaders and public officials in his defense."10 Matatandaang ang McCarthyism ay halaw sa pangalan ni Senador Joseph R. McCarthy ng Kongreso ng Estados Unidos na nagpasimuno ng mga imbestigasyon hinggil sa di-umanoy Un-American Activities noong dakong huli ng dekada '40. Ito ay bungsod ng pagkakatuklas na ang dating bansang Unyon Subyet ay nakalikha na rin ng sandatang atomika at ito ay dahil sa paggamit nila ng mga espiya at secret agents na gumagala sa Amerika. Humantong ito sa isang mapinsala at laganap witch-hunt na nagbungsod lamang ng maraming pasakit sa mga inosenteng tao na napaghinalaang tumutulong o nakikisalamuha sa mga komunista. 1966), 9.

${ }^{10}$ Epifanio San Juan, Rice grains : Selected poems (New York : International Publications, 
Bilang epekto ng mga pangyayaring ito sa Estados Unidos ay itinatag sa Pilipinas ang CUFA o Committee on Un-Filipino Activities. Sa isang panayam noong 1949 kay Amado Hernandez bago pa siya makulong at maparatangang komunista ng pamahalaan ay ganito ang kanyang sinabi tungkol dito:

Isang malaking katuwasan ang nangyari sa Pilipinas. Pagkatapos ilagda ng pamahalaan ang pangkalahatang amnistia na nagpapatawad sa kasalanan at kaparusahan ng mga maniki, taksil, espia, ahente sa buy and sell ng Hukbong katihan at Hukbongdagat ng Hapon, at sa lahat ng tumulong sumaklolo at nagdulot ng kaluwagan sa kaaway, ang pamahalaang ding ito ay lumikha ng CUFA upang usigin ang mga lider ng paggawa, magbubukid, mga tunay na makabayan at mga intelektual na progresibo, na sa kanilang maningning na rekord sa panahon ng digma at kapayapaan ay nagpatunay nang walang alinlangan na higit silang makabayan kaysa mga sa kanila'y tumutuligsa at nag-uusig .

Ang rekord ng CUFA ay ito: Sa loob ng isang taon ay nakagugol nang P 100,000.00 salapi ng bayan, nakapaglathala ng isang report na may 72 pahina na tumuturol sa tatlong komunistang Pilipino (Balgos, Capadocia at Taruc) na sa simula pa ay talaga naming lantaran na sa kanilang panananlig sa Komunismo, hindi pa kamalakmalak na itatag ang CUFA. ${ }^{11}$

Nakapagpiyansa si Amado Hernandez noong Huly 20, 1956, anim na taon matapos siyang ikulong. Taong 1964, Mayo 31 nang siya ay mapawalangsala ng Korte Suprema.

Sa kabila ang hirap sa buhay piitan, nagawa ni Hernandez na ipagpatuloy ang kanyang pagsusulat. Sa loob ng halos anim na taong pagkakabilango, sinulat niya ang mahigit na 40 tula, isang tulang -kasaysayan Bayang Malaya, ang nobelang Luha ng Buwaya, at ang balangkas at panimulang sulat ng nobelang Mga Ibong Mandaragit. Ayon kay Teodoro Agoncillo sa kanyang paunang salita sa obrang Bayang Malaya, "Lubhang malaki ang nagawa ng bilangguan kay Amado V. Hernandez. Ito'y hindi matatawaran. Sa bilangguan namatay si Amado V. Hernandez na makata ng mga pangarap, ng mga buwa't bituin, ng mga bulaklak at paru-paro, ng mga dalaginding at talubata. At sa bilangguan ding iyan iniluwal si Hernandez na makatang palaisip, maka-manggagawa, makatarungan, at makalipunan."12 Hindi iilan sa mga akda ni Ka Amado nang mga panahong ito ang naglalarawan sa hirap ng buhay-piitan. Tulad halimbawa ng tulang "Ang Aking Panauhin," tungkol sa isang preso na nagkaroon ng pangitain ng kanyang ina na nangangaral sa kanya

\footnotetext{
${ }^{11}$ Hernandez, Progressive Philippines, 22-23.

12 Amado V. Hernandez, Bayang Malaya (Quezon City: ADMU Press, 1969), xv.
} 
at nagbibigay ng lakas ng loob. Ito ay isang paggunita ni Hernandez sa kanyang ina na pumanaw noong Hulyo 9, 1953, habang siya ay nakakulong sa bartolina ng Muntinlupa. Ang tulang "Isang Dipang Langit" ay naisulat sa loob ng bartolina noong Abril 22, 1952. Sa tulang ito na itinutiring na pinakamahusay sa mga isinulat ng autor ay inihayag ni $\mathrm{Ka}$ Amado na ang kulungan ay hindi lamang isang parusa, ito ay isang paraan upang ipiit at siilin ang kanyang isip at diwa, pati na rin nang bayan. Subalit hanggat siya ay walang takot at hindi nawawalan ng pag-asa, siya ay mananatiling malaya.

Ako'y ipiniit ng linsil na puno

Hangad palibhasang diwa ko'y piitin,

Katawang marupok, aniya'y pagsuko,

Damdami'y supil na't mithiin ay supil.

Ikinulong ako sa kutang malupit;

Bato, bakal, punlo, balasik ng bantay;

Lubos na tiwalang sa buong daigdig

At inaring kahi't buhay man ay patay.

Sa munting dungawan, tanging abot-malas

Ay sandipang langit na puno ng luha,

Maramot na birang ng pusong may sugat,

Watawat ng aking pagkapariwara. ${ }^{13}$

Ang miserableng sitwasyon sa bilibid na madamdaming inilarawan sa tulang ito ay makikita rin sa maikling kwentong "Panata ng Isang Lider." Tungkol ito sa karanasan ni Noel, lider ng mga manggagawa, nang siya'y ipakulong dahil sa paratang na utak sa pagkakasunog ng isang bahay-kalakal. Inihambing ni Hernandez ang kulungan sa isang impiyerno, kung saan masasaksihan ang kapalaluan, pagsasamantala, kasakiman, at kalupitan ng tao sa kanyang kapwa. Ang bilangguan ang isang sagisag ng kawalan ng katarungan, kung saan ang mga napipiit ay mga taong hindi sapul na masama, bagkus sila ay pawang mga kulang palad na nakulong sa maling paratang o kung nagkamali man, ito ay dala na rin ng kanilang kapaligiran o katayuan sa buhay. Subalit higit ang pasakit ng asawa ni Noel na si Marta na napilitang maghanap-buhay at magsanla ng ariarian upang matustusan ang pangangailangan niya at ng kanilang bugtong na anak, pati na rin ang mga gugol sa demanda. Ito rin ang naging kapalaran ng asawa ni Amado at ng kanyang anak na si Rene. Si Atang dela Rama, na isang mutya ng teatro na namumukod noong dekada ' 20 - ' 30 , ay napilitang isangla ang kanyang bahay at ari-arian upang matustusan ang gugol sa kasong legal ng kanyang asawa.

Ang karanasan ni Noel sa bilangguan, ang kanyang mga , ang mga katotohanang nakita't nakilala niya, ay higit na

${ }^{13}$ Hernandez, Isang Dipang Langit, 63. 
nagpatigas sa kanya, sa kanyang puso, sa kanyang kaluluwa, sa kanyang panananlig. Datapwat hindi siya tumigas ng katulad ng isang bilanggong sa kawalang-pagasa'y napoot sa kasamaan ng daigdig at namatay ang damdaming makatao. Manapa'y nagkaroon siya ng tibay at panata ng santo sa gitna ng mga tukso at ng isang bayani sa gitna ng mga panganib. Kung papupurihan si Noel, masasabing hindi siya nagawang sirain ng makapal na pader at masinsing rehas na bakal. Ang kompirmasyon o pagpapatibay sa kanyang namumukod na pagkatao ay naganap sa bilangguan. ${ }^{14}$

Sa Bayang Malaya mamamasdan ang "puso't kaluluwa ni Hernandez, ang mithiit pangarap ng anakpawis, ang dalamhati't kalagayan ng nagdaralita, ang tigib na kasakiman ng makapangyarihan, ang pag-ibig at buhay ng mga mapangarapin, at ang pag-asa't pag-asam ng bayang Pilipinas." 15 Tulad ng mayakda, ang pangunahing tauhan na si Lantay ay pinuno ng mga manggagawa na nagdusa sa piitan dahil sa maring paratang at baluktot na sistema ng katarungan.

Ipiniit ako noon ng Hapones,

Laksang katulad ko ay laong napiit; Pagka't sa panahong

Ang nasa dambana ay bangis at lupit Walang panginoong

Magbibigay-laya sa sigaw ng matwid. (4896)

Nang kakastilaan, baya'y taga-amen

At hinahagkan pa ang kamay ni Kain, Pag may tumuligsa

Sa gawa ng kura't punong mapaniil, Isang kawang-gawa

Ang siya'y ipangaw na lamang sa karsel. (4902)

Maging sa panahon ng Amerikano,

$\mathrm{Na}$ ang Pilipinas ay sa Pilipino,

Habang ang sakristan

Ay sa Amerika paroo't parito Ay may bilangguan

Sa balang kaaway ng imperyalismo. (4908)

14 Amado V. Hernandez, Langaw sa Isang Basong Gatas at iba pang mga Kwento (Quezon City: UP Press, 1996), 404.

15 Teodoro Agoncillo, in Hernandez, Bayang Malaya, xi. 
Mariing nilinaw ni Hernandez na ang pagkakakulong sa kanya ay isang politcal harassment ng kanyang mga kaaway sa lipunan. Ang mga bintang ay pawang walang katotohanan at gawa-gawa lamang upang pigilan siya sampu ng kanyang mga kasama na ipaglaban ang hustisya sosyal. Sa tulang Bayang Malaya, isinaad ni Lantay sa kanyang liham kay Tala kung paano siya pinakulong dahil sa maling paratang na siya ang nagsulsol sa mga tao upang maging maligalig at mag-aklas laban sa estado. Winika ni Lantay na ang anumang rebolusyon ay bunga, hindi ng anumang pagsusulsol, kundi ng kahirapan, pagmamalabis ng maykapangyarin, at bulok na lipunan. Ngunit sa halip na tugunan ang mga karaingan ng mga mahihirap, ang ginagawa ng maykapangyarihan ay inuusig at sinusugpo ang mga lider ng mga kilusan upang maiwasan ang anu mang pagbabago at mapanatili ang status quo. Gayon pa man, hindi siya nawalan ng pag-asa na siya ay mapapawalang sala sa bandang huli.

Inihawig ni Hernandez ang kapalaran ng pangunahing tauhan na si Lantay, hindi lamang sa kanyang sariling buhay kundi sa kapalarang sinapit ng bayang Pilipinas. Tulad ni Lantay, ang Pilipinas ay naghirap sa kamay ng mga dayuhan. Ang pagkakaalipin ng Pilipinas sa kamay ng dayuhan ay masusing tinalay ni Hernandez sa kanyang mga kolum sa magasin at sanaysay.

Matagal nang panahong ang mga palsipikado ay siyang nasa tanghalan, sa dambana at pedestal. Sila ang gumagawa ng mga batas sa kanilang kabutihan at hindi sa lahat. Sila ang nag-uutos at nagpapatupad ng mga batas para sa maliit. Lambat ng panghuli ng mga sapsap. Samakatuwid, sapul sa panahon ng Kastila hanggang sa panahon ng Kano, nabago lamang ang panahon at ang panginoon, ngunit hindi ang kalagayan, ang sistema, ang sustansiya ng mga bagya-bagay. ${ }^{16}$

Tulad halimbawa ng kwentong "Ang Aguinaldo ng Kanyang Anak" kung saan si Nestor ay napilitang huminto sa pag-aaral nang makabangga niya ang isang propesor na Amerikano. Sa "Langaw sa Isang Basong Gatas," inilarawan ni Ka Amado ang pagiging iskuwater ng mga Pilipino sa kanilang sariling bayan habang ang mga mayayamang dayuhan ay nakatira sa malaki at ekslusibong subdivision. Sa kabanata 24 ng Luba ang Buwaya, walang makitang karehang Pilipino sina Bandong at Pina nang lumuwas sila sa Maynila. Lahat ng restawran, pansiterya, represmen, at kitsinet ay ari ng dayuhan. Ang ating ekonomiya ay kontrolado ng dayuhan sa ano mang panahon o kadahilanan, ang mga Pilipino ay naninilbihan lamang, alipin na maituturing sa kanilang sariling bayan.

Bukod sa mga Amerikano at Hapon, prominente sa mga katha ni Hernandez ang pagmomonopoliya ng mga Intsik.

${ }^{16}$ Herandez, Magkabilang Mukha ng Isang Bagol, 143. 
Ang kapangagaw ng Hapon ay ang mga komersiyanteng Intsik na halos siya namang may monopolyo sa ating mga tablerya, parmasya, panaderya, otel at restawran. Kanila ang sigarilyong lokal. Kanila ang naglalakihang gusali sa purok ng negosyo, ang mga bodegang imbakan ng bigas at asukal, at pinapasok na nila ang mga sine at transportasyon. Ang Intsik ay siya pa ring namamayani sa mga tindahang tingian, sa kabila ang sinasabing batas sa nasyonalisasyon ng mga tindahangbayan.

Samakatuwid, ang mga Pilipino ay parang naka-ipit sa dalawang bato, sa Hapon sa mga negosyong galing sa labas, at sa Intsik sa mga bagay-bagay na yari sa Pilipinas. ${ }^{17}$

Sa nobelang Luba ng Buwaya, nakikipagsabwatan ang mga Intsik sa mag-asawang Grande sa pang-aabuso sa mga mahihirap na magsasaka. Si Dory ("Hubad"), ay isang tindera ng sweepstakes na pinagnasahan ng kanyang dating employer na Intsik na nagmamay-ari ng pabrika ng biskuwit. Isang Intsik din na nagmamay-ari ng naytklab sa kamaynilaan ang tumukso at nagmulat kay Fidel, isang bagong halal na alkalde, sa larangan ng korapsyon sa kwentong "May Uling ang Palayok" (1959).

Subalit nilinaw ni Hernandez na ang kanyang hangad ay ipaglaban ang interes ng mga Pilipino. Ang kanyang pagka-Pilipino ay hindi tuwirang antiAmerican o anti-foreign. "Walang sino mang tunay na unyonista na nagtataglay ng damdamin laban sa alin mang lahi.... Hindi ako naniniwala sa pangingibabaw ng alin mang lahi at kinasusuklaman ko ang pagtatangi sa lahi."18 Ang kanyang lantad na pagsalungat sa imperyalista at pasistang pagtrato ng ilang dayuhan sa kanyang mga kababayan ang naging ugat nang pag-usig kay Hernandez at iba pang lider ng kilusang magpapalaya. Sa tulong ng CIA, militar, at iba pang tuta ng makapangyarihang dayo, ang mga hunyangong Pilipinong politiko ay nagakusa sa kanya bilang komunista at kaaway ng bayan at demokrasya. Ang kanyang karapang pantaong sinasagutan ang konstitusyon ay niyurakan.

Sa panayam na nabangggit noong 1949 bago pa siya ikulong sa paratang na rebelyon, mariin niyang itinanggi na siya ay komunista. Hindi siya kasapi ng Partidong Komunista ng Pilipinas (PKP) at hindi kailan man nakibahagi sa pagsulong ng layunin ng partido o mga grupong kaalyado nito. ${ }^{19}$

17 Ibid., 174.

${ }^{18}$ Hernandez, Progressive Philippines, 32.

19 Malaki ang naisakripisyo ni Ka Amado at ng kanyang pamilya dahil sa maling paratang na siya ay isang komunista. Sa harap ng Korte Suprema ay napatunayan ni Hernandez na siya ay walang anumang ugnayan sa Partido Komunista ng Pilipinas. Sa mga nagnanais mabasa ang mga argumento at kontra-argumeto sa kasong ito, sumangguni sa People of the Philippines vs Amado V. Hernandez, et al G.R. Nos. L-6025-26 July 18, 1956. Bagamat mahalaga ang naging impluwensya ni Hernandez kay Jose Maria Sison, ang nagtatag ng Communist Party of the Philippines (CPP) noong 1968, at ang kanyang mga akda ay naging popular sa mga tagasunod ng ideolohiyang Marxismo sa bansa, ang mga datos na ito ay dapat isaalang-alang. 
Para sa kanyang mga kaaway, ang sino mang humihingi ng mabuting batas o katarungan ay inakkusahan ng pagiging komunista upang sila ay masiil at maipakulong. Ang komunismo ay hindi supling ni Lenin o Stalin, ito ay bunga ng laganap na karalitaan, gutom, at sakit na dala ng kasakiman ng tao. Tutol siya sa sistemang komunismo - sa bayang malaya ay walang komunista - wika ni Ka Amano. Salungat din siya sa ano mang uri ng radikal na rebolusyon na gumagamit ng dahas upang magkaroon ng dagliang pagbabago sa lipunan. Ang madugong Bolshevik na rebolusyon sa Rusya na tinitingala ng mga Pilipinong komunista ay hindi makabubuti sa bansa. 20 Tulad ng isang gubat na hindi dapat sunugin upang puksain lamang ang ilang ahas, ang pagbabago'y hindi kinakailangan ang ganap pagwasak sa kabuuan ng sosyedad. Gaano man pilitin ay hindi mabubuo ang isang perpektong lipunan. Subalit hindi ito nangangahulugan na dapat tayong mawalan ng pag-asa at tiisin na lamang ang kamalian. Hindi rin tama na maghintay na lamang ang mga tao na bumagsak ang sistemang kapitalismo upang magsagawa ng pagbabago. Tungkulin ng lahat na Pilipino ang magkaisa at ipaglaban ang kalayaan ng bayan laban sa mga dayuhan at pang-aalipin ng uring may-ari sa pamamaraang mapayapa. Si Ka Amado ay hindi sang-ayon sa paggamit ng dahas, ang lakas ng katwiran at utak ang gamit niyang kalasag. Upang mabago ang lipunan, kailangan munang baguhin ang kaisipan ang mga ordinaryong mamamayan. Ang ano mang uri ng rebolusyong gagamit ng dahas ay magtatapos sa isang uri rin ng paniniil, kung ito ay walang kakambal na gawaing turuan ang mga mamamayan at imulat ang kanilang kaisipan upang makuha ang kanilang pag-panig at suporta. Kapag nagkaisa ang pangkalahatang masa, walang sino man (kahit pa sinong diyos o bathala) ang makapipigil dito na isakatuparan ang ano mang layunin.

Ipinalalagay ko ang balangkas na sosialista, kung itatatag sa malaya at pasulong na Pilipinas ay makalulutas sa maraming malulubhang suliranin ngayon. Ang Feudalismo ay bulok na at sa mga bansang sakop na lamang nakapananatili, pagpasok ng ika-20 siglo, sapagkat akma sa hangarin ng mga kapangyarihang dayuhan sa mga lupaing naturan. Ang kapitalismo, sa kanyang kalagayan ngayon, ay tiyak na lipas na at hindi na agpang sa kasalukuyang katayuang ekonomiko. Bagaman ang balangkas na ito ay marami pang matapat at matalinong tagapagtaguyod, ay isa na lang hagdang bahaghari ng isang maliit na pulutong sa pagakyat sa kapangyarihan, kayamanan, at mga kaluwagan, ngunit patuloy namang nagpapamulubi sa mga nakararami sa mga mamamayan. Kung ito'y dumating sa sukdulan ang ganyang

20 "The two dates - 26 August and 7 November - are "sacred" to Filipino communists. Evidently the communist founding fathers had so designed that the CPP should symbolize the spirit of two significant revolutions in our time: the Philippine and Russian (Bolshevik) Revolutions." Alfredo Saulo, Communism in the Philippines: An Introduction ( Quezon City: ADMU Press, 1990), 2. 
kapangyarihan ay nagiging Pasismo na angkop sa tawag na 'Diktadura ng Malalaking Negosyo.'21

Mahusay na inilarawan ni Ka Amado ang hindi pagkakapantay-pantay sa kalagayan sa buhay ng mga Pilipino sa kanyang mga obra. Tulad halimbawa ng Luba ng Buwaya kung saan tinalakay ang problemang agraryo sa Pilipinas. Ang mga mahihirap na magsasaka na nagbubungkal ng lupa ay walang lupa at lalong naghihirap samantalang ang mga may-ari ng lupa ay lalong yumayaman. Wika ng isang tauhan sa nobela na is Ba Inte:

Ang lupaing ito'y dati ring gubat na hinawan ng ating mga magulang... Binungkal at pinagyaman nila. Samantalang ang mga lumitaw na mi-ari nang pakikinabangan na ang lupa, buhat sa mga prayle hanggang sa mga Grande'y siyang nagtamasa nang katakut-takot. Ang tanging minana nati'y ang paninilbihan. At kung naisin nating makawala sa gayon paniniil, ang mapapalay bawian ng saka at itaboy. Ewan ko, kung hanggang sa'n pa ang maipagtitiis ng 'sang mahirap. ${ }^{22}$

Bukod sa pangangamkam ng lupa at "Katiwalian sa mga rehistro ng lupa na lumikha ng alingasngas at sigalot lalo na sa Kamindanawan at sa mga lugal na dinarayo ng maraming nagbukas na bomestead,"23 pinaksa rin ni Hernandez ang problema ng mga mahihirap na magsasaka na umaasa lamang sa mga maylupa sa lahat ng kanilang mga pangangailangan sa pagtatanim dahil sa kawalan ng kooperatiba o tulong sa gobyerno. Sila tuloy ay nalulubog sa utang na humahantong sa tinatawag na bili-sanla ng kanilang sakahan. Makikita rin natin ang hindi patas na areglo ng maylupa at mga kasama sa hatian ng ani tulad ng sistemang takipan at talinduwa na lalong nagpapahirap sa kanila. Ang lahat ng ito ay nagaganap sa harap ng "balisawsawing Land Reform ng gobyerno." 24 Ganyan din ang larawang-lipunan na ipinakita sa atin ni Ka Amado sa Bayang Malaya. Patuloy ang kaapihan at paghihirap ng mga Pilipino matapos ang ikalawang digmaang pandaigidig sa kamay ng mga makapangyarihang uri. Ang malaking agwat na ito sa ating lipunan ay hindi makabubuti sa ating lahat, ito ang magiging sanhi ng ating pagbaksak, tulad ng isang gusaling itinayo sa buhangin.

Ang karalitaa'y di nating tadhana,

Iya'y gawang tao na kasumpa-sumpa

Angkan, lipi, sali'y

Dayukdok sa isang lupaing sagana

${ }^{21}$ Hernandez, Progressive Pbilippines, 20.

22 Amado V. Hernandez, Luba ng Buwaya (Quezon City: ADMU Press, 1983), 168.

${ }^{23}$ Ibid., 194.

${ }^{24}$ Hernandez, Magkabilang Mukha ng Isang Bagol, 128. 
At buhay-alipin

Pati bunso't anak sa bayang malaya. (4236)

'Mga komunista' pag nagyakapyakap

'mga komunista' pag nagsiaklas, 'mga komunista'

pagka humungi ng kaunting dagdag, 'mga komunista'

pagka humingi ng mabuting batas. (4428)

Bisig na nagsaka'y siyang walang palay, Nagtayo ng templo'y siyang walang bahay; Dumungkal ng mina

$\mathrm{Ng}$ bakal at ginto ay baon sa utang; Lingkod sa pabrika

$\mathrm{Ng}$ damit ay hubad ang mahal sa buhay. (4470)

Nguni't pag sa lagay ng buhay hinati

At ang panuntuna'y umari't magtangi Iya'y pagtatayo

Sa lupang buhaghag ng isang gusali, Sabay na magluto

$\mathrm{Ng}$ ulam-sinaing sa isang kawali. (4938)

Ang kwento ng Bayang Malaya ay umiikot sa isang barrio na pinangalanang Pinagbangunan. Sa simula, ito ay mistulang paraiso na mapayapa at tahimik, kung saan kahit sa hatinggabi, ang mga pintuan ng bahay ay hindi nakasusi. Ang mga nakatira, bagamat salat sa salapit at luho sa buhay ay may busilak na puso at likas na kagandahang asal. "Hindi isang pook lamang ang inilarawan ng makata, kundi ang nayon ng Pilipinas - ang pinakapuso ng buhayPilipino. Ang Pinagbangunan, bilang kalulwa't bisig ng bayan, ay isang pook na hindi naabot ng malikot at salawahang kabihasnan at samakatwid ay pook na lantay at walang pagkukunwa." 25 Mula sa pagiging paraiso, ang bayang ito'y unti-unting nabulok sa pagdating ng mga Hapon at pagkatapos ng digmaan, sa mga pahirap ng mga kapitalista at dayuhang monopolista. Ang pangunahing tauhan na is Lantay ay naging lider ng anakpawis at nakulong dahil sa maling paratang. Sa kanyang liham kay Tala na kanyang kasintahan, mababakas ang takipsilim sa kalagayan ng bayang mistulang impiyerno, isang takipsilim na may bahid ng kaunting liwanag.

Nakita kong ganap ang kalunos-lunos

$\mathrm{Na}$ hirap ng madlang ayaw pabusabos, Angaw ang nasawi

Sa punlo, sa sable, piitan at bundok,

25 Agoncillo, in Hernandez, Bayang Malay, xi. 
At sa aking budhi

Isang kamatayan ang bawa't himutok. (4998)

Nangangalagas silang wala man ni bahid

$\mathrm{Ng}$ panghihinayang o ng pagkahapis

May taglay pang tuwa

Sapagkat ang alay sa bayang inibig

Ay lubhang dakila

Isang walang hanggang pagpapakasakit. (5004)

Pupong kamatayan ang aking hinamon

Tao, kalikasan, apoy at patibong

Sa Fuerza Santiago

At sa Sierra Madre araw-gabi'y naron, Tulad ng anino

$\mathrm{Na}$ di iiwan hanggang sa kabaong. (5010)

Saan patutungo ang bayang Pilipinas? Para kay Ka Amado, ang tanging solusyon sa ating mga suliranin ay nasa kamay ng mga Pilipino na rin. Hindi tayo dapat umasa sa mga dayuhan o sa pamahalaan. Kailangan tayong magkaisa, ibigay ang ating buong kakayahan upang ang bawat isa ay magkaroon ng karampatang biyaya. "Ang kailangan ng Pilipinas ay ang bayan na rin - mga estudyante, manggagawa, magbubukid, mga tapat na intelektuwal at mulat na karaniwang tao - na siyang magtitindig ng bagong bansa sa sinapupunan ng pagkakaisa, kalayaan, kaunlaran, at hustisya sosyal."26

Sa kwentong "Supling ni San Husep" at nobelang Luha ng buwaya, nagkaroon ng pagbabago sa buhay ng mga magsasaka nang magsimula silang mag-organisa at ipaglaban ang kanilang mga karapatan. Inilahad ni Hernandez sa mga akdang ito na "Hanggang sa barrio'y naiintindihan na ngayon ang hustisya sosyal." 27 Sa tulong ni Bandong, nagkaisa ang mga iskuwater sa tambakan na magtulungan upang maiangat nila ang kanilang kalagayan sa buhay.

Naniniwala si Hernandez na ang sinasabing malayang pangangalakal o free enterprise ay humahantong lamang sa monopoliya. Ang kailangan ng bayan ay nasyonalisasyon ng mga public utilities at ng malalaki at importanteng korporasyon. Kailangan din ang modernisasyon at industrialisasyon upang tayo ay makagawa ng mga produktong ating kailangan sa halip na angkatin ang mga ito sa ibang bansa. Ang karapatan ng mga manggagawa ay dapat pangalagaan at irespeto. Ang kanilang pangaraw-araw na sahod ay dapat maging sapat sa kanilang pangangailangan. Higit niyang ikinalungkot na ang pangkaraniwang trabahador ay sumasahod lamang ng apat na piso isang araw samantalang ang kanyang pang-araw-araw na gastusin ay umaabot sa anim na piso kada araw. Sa

${ }^{26}$ Hernandez, Magkabilang Mukha ng Isang Bagol,132.

${ }^{27}$ Hernandez, Langaw sa Isang Basong Gatas at iba pang mga Kwento, 364. 
madaling salita, dapat ipatupad ang tunay na hustisya sosyal upang maitaguyod ang pagkakapantay-pantay ng lahat ng uri.

Sa Luba ng Buwaya, hiniling ng mga magsasaka kina Don Severo at Donya Leona ang mga sumusunod:

1. Kilalanin ang samahan ng magsasaka.

2. Pagpataw ng interes sa kanilang mga utang sa halagang itinatakda ng batas.

3. Sarilihin ng maylupa ang gugol sa binhi, yamang ang binhi'y bahagi ng puhunan.

4. Pagkatapos ng bawat aniha'y magkaloob ang maylupa ng sapat na pataba upang magamit sa susunod na pagtatanim.

5. Pautangin ang kasama sa panahon ng pagtatanim at dalawang buwan bago maggapas ng halagang ang kabuua'y hindi lalampas sa kalahati ng mga magbabahagi.

6. Ituring ang mga magsasaka na tao at hindi alipin.

7. Ipatupad ang tunay na repormang agraryo.

Wika nga ni Ginoong Tasyo sa nobela: “ang hinihingi po nami'y hindi habag, ni kagandahang loob. Ang hinihingi po nami'y hustisya. Ang habag at kagandahang loob e kabutihang galing se'nyo. Samatalang ang hustisya po'y amin, at talagang karapatang hindi dapat itanggi." 28

Para kay Hernandez, ang sosyalismo ay hindi Marxismo o ateismo:

Socialist economy was practiced by men of good will thousands of years before the discovery of Marxism. Says the Acts in the New Testament: "All that believed were together, and had all things common; and they sold their possessions and goods, and parted them to all, according to any man had need. Catholics call it Christian Communism. ${ }^{29}$

Muli, sa nobelang Luha ng Buwaya, ipinagtanggol ni Atty. Reyes ang karapatan ng mga iskuwater na magkaroon ng lupa sapagkat ito ay alinsunod sa Banal na Bibliya:

Tumakas ang mga Israelista sa kalupitan ng Paraon, at humanap ng bagong lupain sa pangunguna ni Moises... Humantong sila at humimpil sa mga kapatagan sa kanluranin ng Ilog Nilo, sa labas ng mga hangganan ng Ehipto. Walang nagsabi sa kanilang ang lupang iya'y akin, at pinagpala sila ni Hehoba. ${ }^{30}$

${ }^{28}$ Hernandez, Luha ng Buwaya, 216.

${ }^{29}$ Hernandez, Progessive Philippines, 6.

${ }^{30}$ Hernandez, Luba ng Buwaya, 31. 
'Tulad ng iba pa niyang mga akda kung saan ginamit niya ang tauhan upang maihayag ang kanyang kaisipan, tinukoy ni Ka Amado ang mga turo ng dakilang pantas tulad nina Platon at Santo Tomas de Aquino hinggil sa pagaari.

Mababasa n'yo sa Republika ni Platon ang hangad niyang komunismo sa mga lupain at asawa, na bagaman ito'y sinalansang ni Aristoteles nitong huli'y iminungkahi naman ng tagapayo ni Alehandro Magno na lagyan ng takda ang mga ari-ariang partikular na maaring lagumin ng isang tao.

Sa panahon ni Thomas Moore, gunita ng manananggol, nang lumabis ang kasakiman ng mga pinunong piyudal, na ngayo'y siyang katimbang ng ating mga uldog, sinulat ni Moore and kanyang "Utopia" kung saan ang lupa'y pag-aari ng buong lipunan at ang paghahati sa mga kailangan ng tao'y bataty sa pagkakapantay.

Ipinaalala niyang utang sa dalawang paraan ang ipinagkaroon ng sariling kanya ng isang tao.

Una'y sa kanyang kasipagan at pag-iimpok, ani Abugado Reyes. Ikalawa'y sa kanyang kasakiman at panghahamig. Ngunit madaling makilala kung sino ang nag-impok at kung sino ang nanghamig, gaya ng pagkilalas sa kaibhan ng bangus at ng pating.

Ipinaalala ng manananggol ng mga iskuwater na ang lupain, kayamanan at iba pang biyaya'y hindi wastong sarilihin at pag-imbutan ng mga nag-iingat, patkat sila'y mga katiwala lamang. Tinukoy niya ang paninindigan ni Santo Tomas Aquinas, isa sa mga pangunahing diwa ng Katolisismo, na ang kayamanan at ari-ariay hindi pansarili ng naghahawak, kundi dapat gamitin sa kabutihan ng madla. ${ }^{31}$

Si Hernandez ay hindi kailan man nawalan ng tiwala sa sistemang demokrasya. Taliwas sa pananaw nina Jose Lava o Luis Taruc na nag-nanais sirain ang sistemang kapitalismo bago magsagwa ng mga reporma, mas makabubuti para kay Hernandez na isagawa ang mga reporma sa loob ng sistemang kapitalismo mismo, sa paggamit ng demokratikong proseso. Batid ni Hernandez ang kahinaan ng ating pamahalaan at ng sistemang demokrasya sa bansa. Sa kanyang mga sanaysay (1969-1970) ay pinuna niya ang mga korapsyon, labis na paggugol sa halaan, dayaan sa halalan, nepotismo at daynastiya sa gobyierno, at iba pang katiwalian sa ating pamahalaan. Ang mga

${ }^{31}$ Ibid., 317. 
pangunahing tauhan sa ilan sa kanyang mga katha tulad nina Lantay (Bayang Malaya), Bandong (Luba ng Buwaya), Andres ("Isang Ulo ng Litsyon"), Noel ("Panata ng Isang Lider") at Tonyo ("Supling ni San_Husep") ay pawang mga biktima ng maling paratang at kawalan ng katarungan. Ang pamahalaan ay nasa kamay ng mga mayayaman na nais panatiliin ang status quo. Muli, sa Bayang Malaya,

Sa pamahalaa'y ang bayan ang nagtatag,

Ang pamahalaan ay ari ng lahat;

At yamang ang dukha

Ay nakahihigt sa bilang at lakas, Nararapat yata

Na magkaluklukan ng karapat-dapat. (4380)

Ang baya't gobyerno'y kung nagkakasundo

Walang panihala't gawang mabibigo,

Nguni't pagka hindi

Anomang buui'y hindi mabubuo;

Puso't utak wari,

Sa aba ng tao pagka nagkalayo. (4386)

Wala tayong hangad yumurak ng iba,

Mang-agaw ng aring di naman ginaga,

Di tayo kalaban

$\mathrm{Ng}$ yama't puhunang makademokrasya,

Ang ating kaaway

Ay ang kasakima't pagsasamantala. (4518)

Gasino na ako't gasino ang iba?

Kahi't nila kitlin ang isa, dalawa

$\mathrm{O}$ ang ilang bunton

$\mathrm{Ng}$ nasang lipuli'y buhay ang kalulwa;

Hindi malilipol

Ang anak ng bayang may pagkakaisa. (5022)

Naniniwala siya na sa demokratikong pamahalaan lamang may kalayaan at pagtingin sa kapakanan ng nakararami sapagkat sa demokrasya, ang pamahalaan ay pag-aari ng lahat. Kung sa gayon, ang mga mahihirap na higit na nakararami sa lahat ay dapat na magkaroon ng mas malaking puwang sa paglikha at pagpapatupad ng batas. Dahil sa salapi, ang mga maykaya ay nagagawang manipulahin ang batas at kasangkapanin ang estado, subalit ang kainaman pa rin sa demokrasya ay ang pagbibigay nito ng pagkakataon para sa minorya o oposisyon na sumalungat at tumuligsa sa mga nanunungkulan. Sa madaling salita, sa demokrasya ay may kalayaan sa pagpapahayag ng kaisipan, hindi iisang tinig o ideolohiya lamang ang umiiral. Subalit kung ang pagkakahati ng mg tao sa ideolohiya ay makabubuti sa bayan dahil sa naidudulot nitong pagbalanse ng 
kapangyarihan or check and balance, ang pagkakahati ng mga mamamayan base sa kanilang katayuan sa buhay ay makakasama. Upang maging ganap ang ating pagkademokrasya, kailangang ang kapakanan ng nakararaming mahihirap ang dapat mangibabaw at mabigyang pansin. Upang ito ay maisakatuparan, hindi kailangan ng madugong rebolusyon o diktadura ng proletaryo, ang kailangan ay ang pagkakaisa at progresibong pagkilos ng mga mahihirap na higit na nakararami ang bilang.

\section{Pagsusuri at Konklusyon}

Ang sosyalismo ni Hernandez ay maituturing na isang Pilipinismo. Hindi ito isang kaisipang hilaw na hinango sa mga banyaga, hindi likhang isip o kopya sa anumang aklat, ito ay bunga ng kanyang sariling karanasan o lived experience bilang isang manunulat na may mulat na kaisipan at nakikiisa sa laban ng kanyang kababayan upang magkaroon ng tunay na katarungang panlipunan. Sa kadahilanang ito, ang sosyalismo ni Hernandez ay orihinal at personal, hindi sapat na ihanay lamang siya sa mga manunulat na makakaliwa tulad nina Mao, Lenin, o Marx sapagkat iba ang kanilang buhay-karanasan. Di Tulad ng mga pilosopong nabanggit, hindi nagsagawa si Hernandez ng isang pangkalahatan at abstraktong teoriya ng kapitalismo at estado, sa halip ay nagbigay siya sa kangyang mga obra ng detalye at kongkretong paglalahad sa katayuan ng sistemang pulitikal at sosyal na umiiral sa bansa batay sa kanyang naranasan bilang isang manggagawa, peryodista, aktibista, political prisoner, at higit sa lahat, bilang isang Pilipino sapagkat ang kanyang mga pinagdaanan ay hindi naiiba sa mga naranasan ng maraming Pilipino noon man o ngayon. Ang ano mang pagsusuri ng mga sinulat ni $\mathrm{Ka}$ Amado ay dapat itong isaalang-lang. Kung hindi, ang kanyang pananaw ay magiging isa lamang na metapisikal na doktrina na nagbibigay ng solusyong a-priori sa mga suliraning panlipunan. Sa madaling salita, ang sosyalismo ni Hernandez ay hindi isang teoriya, ito'y isang praxis.

Una sa lahat si Ka Amado ay isang makatang Pilipino. Ang kanyang pagiging soysalista ay kakambal ng kanyang pagiging makabayan. Sa kanyang mga akda ay kanyang inilarawan ang mga problemang binabalikat ng mga Pilipino at malalim niyang sinuri ang mga sanhi nito. Ilan sa mga suliraning ito ay ang kahirapan, kawalan ng katarungang panlipunan, problemang agraryo, pagsiil sa kalayaan ng mga manggagawa na mag-organisa (unionism) at ipaglaban ang kanilang mga karapatan tulad ng makataong pagtrato sa kanila ng makapangyarihang kapitalista, makatarungang sahod, hindi lalagpas sa walong oras kada araw na paggawa, seguridad sa pagtrabaho, tamang benepisyo iba pa. Sa pananaw ni Ka Amado, isang sistemang sosyalista ang makatutugon sa mga suliraning ito. Binigyan diin niya ang kahalagahan ng makabuluhang agraryo sa lupa upang maitaguyod ang kapakanan ng mga magsasaka. Pag-siil sa pangaabuso ng mga kapitalista sa karapatan ng pag-aari at kapital ang makatutugon sa mga problema ng mga karaniwang manggagawa. Gayon din ang nasyonalisasyon ng mga malalaking industriya at public utilities upang masupol ang tuluyang pagmomonopoliya ng mga dayuhan at ilang Pilipinong mayayamang kapitalista na sa huli ay nakikipagsabwatan. Ang pagtatatapos ng 
panahon ng Amerikano sa ating kasaysayan ay hindi nagbigay tuldok sa pag-iral ng piyudalismo at imperyalismo, sa halip ay lalo itong lumala. Ang umiiral na pang-aapi ng makapangyarihang uri sa mga mahihirap ay karugtong ng pangaapi ng mga dayuhang mananankop sa mga Pilipino. Ang suliraring agraryo at malaking agwat sa katayuan sa buhay ng mayaman at mahirap ay nag-uugat sa piyudalismong pamamamalakad noong panohon ng kolonyalismo, at naipagpatuloy dahil sa imperyalismong sistema sa ekonomiya kung saan ang mga dayuhan at kanilang interes ang naghahari. Kung gayon, ang class struggle sa Pilipinas ay hindi lamang salungatan ng dalawang uri ng katayuan sa buhay, hindi lamang labanan ng mayaman o mahirap, kapitalista at manggagawa, ito ay nag-uugat sa dalawang magkasalungat na pananaw $O$ ideolohiya: pagkamakabayan at pagiging makadayuhan. Ang proletaryanismo sa bansa ay hindi sapat kung walang katambal na nasyonalismo. Dahil dito ang konseptong class struggle ay hindi sukdulan sa pilosopiya ni Hernandez, kung ikukumpara kay Marx at Engels. Sa Bayang Malaya, binigkas ni Hernandez ang posibilidad na ang puhunan at bisig, kapitalista at manggagawa, ay magyakap at magkaisa, kung iwawaksi ng bawat Pilipino ang pagkamakadayuhan, sa ngalan ng pagiging makabayan. Higit sa lahat, ang pagkakapantay-pantay ng bawat mamamayan sa kalagayan sa buhay ay hindi isang panaginip o pantasya, ito ay isang karapatang pantao. Ang kahirapan ay hindi dala ng tadhana, ito ay sanhi ng kasakiman at pagmamalabis. Kung gayon, ang kahirapan ay kayang lutasin. Ano mang lipunan kung saan malaki ang agwat ng mahirap at mayaman ay hindi magiging tunay na malaya at mapayapa.

Ang sosyalismo ni Hernandez ay makamahirap, makamanggagawa, makadiyos at makademokrasya. Sa lahat ng mga akda, buhay ng karaniwang mamamayan na naghihirap ang kanyang tinalay. Ang pasakit ng mga mag manggagawa ay napakahalaga para kay Hernandez. Ito ay hindi lamang sapagkat higit na nakararami sa mga Pilipino ay kabilang sa uring manggagawa. Ang uring manggagawa ang dugo ng industriya at ekonomiya. Kalulunos-lunos ang kalagayan ng mga maggagawa na kanyang tinawag na "anakpawis" - ang bisig at lakas ng kaunlaran at produksyon ngunit ang katayuan sa buhay ay baon sa utang at salat sa kaginhawahan. Binigyan din niya ng pansin ang mga manggagawa sa agrikultura na sakabila ang henerasyon na pagbungkal sa lupang sinasaka ay nananatiling mga iskuwater sa sariling bayan. Taliwas sa pananaw ni Marx o Lenin, ang kilusang proletaryanismo sa Pilipinas ay walang ganap na kabuluhan kung hindi nito isasaalang-alang ang mga manggagawa sa lupa na higit na nakararami. Ang sosyalismo ay walang kinalaman sa ateismo. Malinaw ang pananalig sa Diyos ni Hernandez, na para sa kanya ay isang Diyos na nagnanais na ang lahat ng nilalang ay magkapantay-pantay at makinabang na tunay sa kanyang nilikha. Sa Bibliya mismo nasusulat ang dakilang turo na ang tao ay katiwala lamang ng yaman ng daigdig pagkat tanging Diyos ang nagmamayari dito.

Ayon kay Hernandez, kung ang sosyalismo man ay magtatagumpay sa Pilipinas, ito ay buntod ng pakikibaka ng mga makabayang mamamayan sa mapayapa at demokratikong paraan. Ito ang dahilan kung bakit pinili niya na gamitin ang mass media tulad ng peryodiko at magasin, at sumulat ng mga 
nobela at maikling kwento, sapagkat sa pamamaraang ito ay maaari niyang maabot ang mas maraming mambabasa, upang gisingin at hubugin ang kanilang kamalayan at makabuo sila ng sarili nilang Weltanschanung. Kung hindi ay mananatili silang parang isang preso na tanging isang dipang langit lamang ang abot-tanaw. Bagamat ang piitan ay isang trahedya, sa kanyang mga tula at kwento ay ipinaliwanag ni $\mathrm{Ka}$ Amado na mas malubhang kamalasan ang pagkakapiit ng isipan at diwa. Toong salat sa elaborasyon at sistematisasyon ang sosyalismo ni Hernandez kung ikukumpara sa pilosopiya nina Marx o Lenin, ngunit sa isang pananaw ay higit na progresibo ang kanayang uri ng sosyalismo. Ang pagbabagong panlipunan ay hindi sanhi lamang ng pagbabago sa ekonomiya o instrumento ng produksyon tulad ng paniniwala ni Marx, ito ay bunga din ng pagbabago sa panananaw at kaisipan ng mga tao. Ang kahirapan at pang-aapi sa mga manggagawa ay hindi sapat upang magkaroon ng isang makabuluhang rebolusyon. ${ }^{32}$ Maaaaring magkaroon ng ganap na pagkakapantay-pantay sa katayuan sa buhay ang mga Pilipino sa pamamamgitan ng mapayapa at demokratikong paraan (si Hernandez mismo ay naging opisyal ng lokal na pamahalaan). Maitutulad ang pilosopiya ni Hernandez sa kaisipan ni Antonio Gramsci (1891-1937), isang Italyanong Neo-marxista at peryodista, itinuturing ng pinakaimportanteng makakaliwang pilosopo ng ika-20 siglo. ${ }^{33}$ Ayon kay Gramsci, ang dahilan kung bakit hindi nagtagumpay ang komunismo o ano mang makamasang kilusan sa Italya ay ang kakulangan sa kamalayan ng karaniwang tao dala ng kawalan ng kultura at literatura na maituturing na nasyonal-popular na bubuuin at itataguyod ng mga organikong-intelektuwal. Kung susundin ang pag-susuring ito, tama si Hernandez hinggil sa kahalagahan ng panitikan sa pagbabago ng lipunan at sa pagsulong ng ideyang sosyalismo.

Malinaw na ang mga suliraning panlipunang nailahad ay hindi lamang masasaksihan sa kasalukuyang panahon, lalo pa itong naging malubha at talamak. Ang mga kasalukuyang mambabasa ng kanyang mga tula at maikling kwento ay wiwikaing palasak na ang kanyang mga konsepto at ang kanyang tema ay bahagi na ng lumang kanta. Sa pagbagsak ng sistemang sosyalismo sa dating Unyong Subyet at mga kaalyado nito, ang mga manunulat na kagaya ni Ka Amado ay itinuturing ng marami na lipas na o laos, sa paniniwalang ang

32 Ang determinista at ekonomistang interpretasyon sa mga sulat ni Marx at Engels ang naghihiwalay sa tradisyonal na Marxismo at Neo-marxismo. Malinaw sa mga akda nina Marx at Engels na binigyang halaga nila ang papel ng ekonomiya bilang base ng lipunan at ugat ng pagbabago. Kung ito may naaayon sa interpretasyong determinista ay maaaring pagdebatihan. Wika ni Friedrich Engels, "According to the materialistic conception of history, the ultimately determining element in bistory is the production and reproduction of real life. More than this, neither Marx nor I have asserted. Hence if somebody twists this into saying that the economic element is the only determining one, be transforms that proposition into a meaningless, abstract, senseless phrase. The economic situation is the basis, but the various elements of the suprastructure - political forms of the class struggle and its results, also exercise their influence upon the course of the historical struggles and in many cases preponderate in determining their form." Karl Marx and Friedrich Engels, On Religion, ed by Reinhold Niebuhr (New York: Shocken Books, Inc., 1974), 275.

33 Maraming pagkakatulad sa buhay at pananaw ng dalawang manunulat. Tulad ni Amado V. Hernandez, si Antonio Gramsci ay isang peryodista, opisyal ng gobyerno at unyonista na nakulong ng mahabang panahon. Batay sa pilosopiyang Gramscean, si Hernandez ay maituturing na isang organikong-intelektuwal. 
makakaliwang tradisyon, ngayon at kailan man, ay wala nang kabuluhan. Masasabing hindi nagtagumpay ang mga akda ni Amado Hernandez na gisingin ang kamalayan ng mga Pilipinong mambabasa at wala itong naging malaking epekto sa pagsugpo sa mga suliraning panlipunan ng bansa. Patuloy ang pagiral ng diktadura ng malalaking negosyo at ang makabuluhang agrayo sa lupa ya malabo pa ring maipatupad. Subalit ang pagkukulang ay hindi kay Ka Amado. Ginampanman niya ang kanyang tungkulin bilang isang makabayang manunulat sa kanyang makatotohanang paglalahad ng kalagayang panlipunan. Ang pagkukulang ay nasa ating mga mambabasa. Nagkulang marahil tayo ng pangunawa sa kanyang mga sinulat, hindi natin binasang mabuti ang kangyang mga akda, o hindi natin ito binigyan ng sapat na pansin.

Department of Philosophy, Bellevue College, United States of America

\section{References}

Agoncillo, Teodoro, A Short History of the Filipino People (Quezon City: UP Press, 1960).

Deveza, Eduardo, A Translation and A Study of Amado V. Hernander's Bayang Malaya (Unpublished Doctoral Dissertation in Literature, University of Santo Tomas Graduate School, 1972).

Hernandez, Amado, Progressive Philippines (Manila: Pilipino Press, 1949) , Isang Dipang Langit (Manila: Makabayan News Feature, 1961). Bayang Malaya (Quezon City: ADMU Press, 1969). Luha ng Buwaya (Quezon City: ADMU Press, 1983).

Langaw sa Isang Basong Gatas at iba pang mga Kwento (Quezon City: UP Press, 1996). 1997). , Magkabilang Mukha ng Isang Bagol,(Quezon City: UP Press,

Marx, Karl, Early Writings, trans. By Rodney Livingstone and Gregor Benton (New York: Vintage Books, 1975).

Marx, K., and Engels, F., On Religion, ed by Reinhold Nieburh (New York : Shocken Books, Inc., 1974).

Pangilinan, Estelita, "A Translation and Critical Analysis of Amado V. Hernandez's Mga Ibong Mangdaragit," Unpublished MA Thesis in Literature, University of Santo Tomas Graduate School, 1976.

Pantoja-Hidalgo, Christina, "History As Literature," Coming Home ( Pasig City: Anvil Press, 1997).

San Juan, Epifanio, Rice grains : Selected poems (New York : International Publications, 1966).

Saulo, Alfredo, Communism in the Philippines: An Introduction (Quezon City: ADMU Press, 1990). 